\title{
Placement Benchmarks for 3-D VLSI
}

\author{
Stefan Thomas Obenaus \\ Scho ol of Computer Sciene \\ McGill University \\ T ed H. Szymanski \\ Communications Research L aborateny \\ McMaster University
}

\begin{abstract}
We pro vide a general defuition of the 3-D wirelength placemert problem. This definition facilitates comparison of 3-D placement algorithms. Wirelength results using partztioning placement are included for the A C.M/SIGDA and ISPD9S standard benchnark circuit suites. Further, a wirelength comparison betw een 2- and 3-D placements is made, and it is shown that larger circuits require $50 \%-60 \%$ less wirelengthwhen utilising the third dimension.
\end{abstract}

Keywords: 3-D, Benchmarks, Placement, VLSI, Wirelength

\section{INTRODUCTION}

As rescarch into three dimensional circuit architectures becomes more widespread (Chiricescu and V ai,1998; Depreitere et al., 1994; Lceser et al., 1998; Leighton and Rosenberg, 1986; Ohmura, 1998; Tong and Wu, 1995), the study of techniques for placing circuits into 3-D structures is emerging. Unlike 2-D placement papers, previous 3-D placement results ha ve been published in isolation (Leeser et al., 1998; Ohrma, 1998) and camot be compared to eac hother for lack of benchmark results. In tw o dimensions wirelengths benbmarks have been a common means of comparing placement algorithms. Howcver, in 3-D no tabulated results for comparison exists. 
Our goal is to fill the void and provide wirelength results for comparison of 3-D placement techniques. For this purpose, we have cxtended the established method of partitioning placement (Shahookar and Mazumder, 1991) to three dimensions. Other common placement methods are simulated annealing and force-directed placement (Shahookar and Mazumder, 1991). We use the currently fastest and best partitioner, hMetis (Karypis et al., 1997). The details of this placement method are described in section 2.. While using a different partitioner, the partitioning placement technique has already been used for 3-D placement for the Rothko architecture (Leeser et al., 1998).

Partitioning placcment is fast and has traditionally yielded good results (Shahookar and Mazumder, 1991). For large circuits, which, as we show in section 3.2, profit most from 3-D technology, an asymptotically fast placement algorithm is essential. Asymptotically slower techniques: such as simulated annealing, are not likely to producc high quality results in an acceptable period of time for these circuits as the cooling schedule would have to be drastically shortened.

Given the high quality of the partitioner, and the fundamental soundness of the partitioning placement techmique, we have confidence that the placement results presented here provide a good benchmark against which future 3-D placement techniques can be measured.

\subsection{PLACEMENT MODEL}

In order to provide a convenicnt basis for benchmark comparisons, the placement model should reflect reality, yet be general and easy to implement. In two dimensions, the Checkerboard model (Shahookar and Mazumder, 1991), a.k.a. the Gute Array Model, serves this purpose. In this model, circuit elements are of identical size and placed in checkerboard-fashion onto a grid. We extend this model to three dimensions in the natural way. While the purpose of this model is to facilitate comparison of placement algorithms, actual placement methods will have much more specific requirements for the grid size and shape, size of circuit elements, pad placements, etc.

The most common measure of quality of a placement algorithm is the total wirelength required for the circuit (Shahookar and Mazumder, 1991). The most efficient method of connecting points, eg. circuit nodes, in space along horizontal and vertical lines using a minimum of wirelength is to construct a rectilinear Steiner tree (Hanan, 1966). 


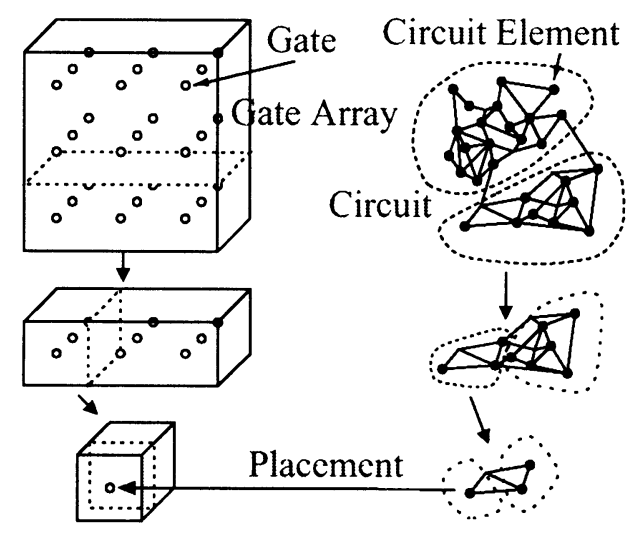

Figure 1.1 Partition Placement Process: Simultaneous Splitting of the Grid and Partitioning of the Circuit

$A$ rectilinear Steiner tree $S(e, f)$ is the shortest tree that connects all nodes $v \in e$ at positions $f(v)$ using only orthogonal segments parallel to the coordinate axes. Its length is $|S(e, f)|$.

However, constructing a rectilinear Stciner trec and measuring its length is difficult. A common approach is to estimate the size of the Steiner trec by adding the width, height, and depth spanmed by the nodes in the Steiner trec. This measure is accurate for two and three node nets. We call this approximation the scmi-perimetcr bounding box approximation $\left|S^{*}(e, f)\right| \approx|S(e, f)|$.

For placement purposes, we reduce all circuit elements to unit-size nodes, and thus we abstract the circuit into a hypergraph $G(V, E)$. $G(V, E)$ is simply the combination of the set of circuit elements, or nodes, $V$, with the set of nets, or hyperedges, $E$, where for all $e \in E, e \subset V$.

It remains to define the 3-D placement model, as a straight forward extension of the 2-D Checkerboard model, for comparison purposes:

\section{Definition 2 3-D Placement Model}

Given a circuit $G(V, E)$ find a reversible function

$$
f:|V| \rightarrow\left\{1, \ldots, n_{1}\right\} \times\left\{1, \ldots, n_{2}\right\} \times\left\{1, \ldots, n_{3}\right\} .
$$

The measure of quality of the placement function is the total wirelength estimate. $\sum_{e \in E}\left|S^{*}(e, f)\right|$.

\section{PLACEMENT METHOD}

Partitioning placement is one of the fundamental placement methods (Shahookar and Mazumder, 1991). When placing a circuit into a two 
Variables and predicates:

$$
\begin{aligned}
& V=\left\{v_{1}, \ldots, v_{|V|}\right\} \\
& x[v] \\
& \left(a_{1}, a_{2}, a_{3}\right) \\
& \left(b_{1}, b_{2}, b_{3}\right) \\
& \left(n_{1}, n_{2}, n_{3}\right)
\end{aligned}
$$

set of circuit nodes

coordinates of gate for circuit node $v$ coordinates of lower left front corner lengths of sides of gate array box initial size of gate array box

Initial Call:

$$
\operatorname{place}\left(V^{r},(0,0,0),\left(n_{1}, n_{2}, n_{3}\right)\right)
$$

$$
\begin{array}{ll}
\text { place } & \left(V_{,}\left(a_{1}, a_{2}, a_{3}\right),\left(b_{1}, b_{2}, b_{3}\right)\right) \\
1: & \text { if }\left|V^{\prime}\right|=1 \text { then } \\
2: & x\left[v_{1}\right]:=\left(a_{1}, a_{2}, a_{3}\right) \\
3: & \text { else }
\end{array}
$$

4: $\quad k:=i$ such that $b_{i}=\max \left(b_{1}, b_{2}, b_{3}\right)$

split box $b$ into two boxes $b 1$ and $b 2$

5: $\quad\left(b 1_{1}, b 1_{2}, b 1_{3}\right):=\left(b_{1}, b_{2}, b_{3}\right)$

6: $\quad b 1_{k}:=\left\lfloor b_{1} / 2\right\rfloor$

7: $\quad\left(b 2_{1}, b 2_{2}, b 2_{3}\right):=\left(b_{1}, b_{2}, b_{3}\right)$

8: $\quad b 2_{k}:=\left\lceil b_{1} / 2\right\rceil$

9: $\quad\left(a 1_{1}, a 1_{2}, a 1_{3}\right):=\left(a_{1}, a_{2}, a_{3}\right)$

10: $\quad\left(a 2_{1}, a 2_{2}, a 2_{3}\right):=\left(a_{1}, a_{2}, a_{3}\right)$

11: $\quad a 2_{k}:=a_{k}+b 1_{k}$

partition $V$ into sub-circuits $V_{1}$ and $V_{2}$

of sizes no nore than $b 1_{1} \cdot b 1_{2} \cdot b 1_{3}$ and $b 2_{1} \cdot b 2_{2} \cdot b 2_{3}$, respectively

12:

$(V 1, V 2):=$ partition $\left(V, b 1_{1} \cdot b 1_{2} \cdot b 1_{3}, b 2_{1} \cdot b 2_{2} \cdot b 2_{3}\right)$

13: invoke placement routine on sub-circuits

14:

$$
\begin{aligned}
& \text { place }\left(V 1,\left(a 1_{1}, a 1_{2}, a 1_{3}\right),\left(b 1_{1}, b 1_{2}, b 1_{3}\right)\right) \\
& \text { place }\left(V 2,\left(a 2_{1}, a 2_{2}, a 2_{3}\right),\left(b 2_{1}, b 2_{2}, b 2_{3}\right)\right)
\end{aligned}
$$

Figure 1.2 Generic Partitioning Placement Algorithm

dimensional gate array, the gates in the gate array are recursively split into smaller sub-arrays. At the same time the circuit is partitioned and the sub-circuits are assigned to the sub-arrays until each circuit clcment can be assigned to its unique gatc. This process extends to three dimensions in the obvious way, as is illustrated in figure 1.1. Figurc 1.2 provides the partition placcment algorithm.

Lecser et al. (Leescr et al., 1998) used a partitioning placement method for placcment in the Rothko architecture. Their partition placement method was based on a 2-D variation of partitioning placement, called quadrisection (Shahookar and Mazumder, 1991). In quadrisection, the chip area is recursively split into four quadrants and circuits are recursively partitioned four ways. They extended this method into three dimensions by splitting the chip's volume into eight octants while concurrently partitioning the circuit eight ways. However, no placement results were published. 


\begin{tabular}{|c|c|c|c|c|c|c|c|c|c|c|c|}
\hline Circuit & Nodes & Vets & Pins & $\frac{p_{1 n u}}{N o d c}$ & $\frac{p_{n+4}}{N_{e t}}$ & Circuit & Nodes & Nets & Pins & $\frac{P_{2 n x}}{\text { Nude }}$ & 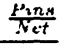 \\
\hline $19 \mathrm{ks}$ & $28+4$ & 3282 & 10547 & 3.71 & 3.21 & s15850P & 10470 & 10383 & 24712 & 2.36 & 2.38 \\
\hline avq.large & 25178 & $25 ; 384$ & 827.31 & 3.29 & 3.26 & s35932 & 18118 & 17828 & 481.15 & 2.6 .5 & 2.70 \\
\hline avq.small & 21918 & 22124 & 76231 & 3.18 & 3.15 & s38417 & 239410 & 23813 & 57613 & 2.41 & 2.12 \\
\hline balup & 801 & 735 & 2697 & 3.37 & 3.67 & s38584 & 20995 & 20717 & 55203 & 2.63 & 2.66 \\
\hline biomedP & 6514 & $5 \pi+2$ & 21040 & 3.23 & 3.66 & s9234P & 5860 & 5844 & 14065 & 2.40 & 2.41 \\
\hline golem3 & $10: 3048$ & 144949 & 3384419 & 3.28 & 2.33 & structP & 1952 & 1920 & 5471 & 2.80 & 2.85 \\
\hline industry2 & 12637 & 13419 & 48158 & 3.81 & 3.59 & $t 2$ & $166: 3$ & 1720 & 6134 & 3.69 & 3.57 \\
\hline industry 3 & 15106 & 21923 & $6 ; 791$ & 4.27 & 3.00 & t3 & 1607 & 1618 & 5807 & 3.61 & 3.59 \\
\hline $\mathrm{p} 1$ & 833 & 902 & 2908 & 3.49 & 3.22 & t4 & 1.51:5 & 1658 & 5975 & 3.94 & 3.60 \\
\hline p2 & 3014 & 3029 & 11219 & 3.72 & 3.70 & t5 & 2.59 .5 & 2750 & 10076 & 3.88 & 3.66 \\
\hline s13207P & 8772 & 86.51 & 20606 & 2.35 & 2.38 & t6 & 1752 & $16+11$ & 6636 & 3.79 & 4.05 \\
\hline
\end{tabular}

Table 1.1 The 1993 ACM/SIGDA Benchmark Circuits

As our partitioner, we chose the hMetis hypergraph partitioner developed by Karypis et al. (Karypis et al., 1997). This partitioner is currently the best and fastest partitioner published. Although w euse recursive tw o-wy partitioning, we could easily implement 3-D quadrisection with a few modifications to the hMetis library interface. Restrictions in the current hMetis library interface made it necessary to compute a recursive balanced $(k+l)$-way partitioning to ac hick a $k: l$ split as is sometimes necessary in step 12 of the algorithm in figure 1.2 when an odd number of rows, columns, or layers needs to be split. While this increases run-time and memory requirement, it does not affect the quality of the cut (Karypis, 1999). According to Karypis, the hMetis in terface could casily be adapted to allow explicit $k: l$ cuts.

In order to compensate for the excessive memory requirement for large $(k+l)$-way partitionings, we restricted the largest dimensions of the gate arra ysfor the largest circuits to be of even length. Consequently, the largest cuts are balanced tw omomory resources. F urther, to obtain accuratc estimates of the rutime, assuming the hMetis in terface $\mathrm{w}$ asadapted to allo w explicit $k: l$ splits, w e ran the algorithm while forcing balanced 2-ray splits at all levels of recursion.

\section{RESULTS}

\subsection{3-D RESULTS}

We performed the benchmark runs on the circuits in the ACM/SIGDA circuit suitc (Brglez, 1993), and the newer ISPD98 benchmark circuit suite (Alpert, 1998), cf. tables 1.1 and 1.2. While the ACM/SIGDA 


\begin{tabular}{|c|c|c|c|c|c|c|c|c|c|c|c|}
\hline Circuit & Nodes & Nets & rins & $\frac{P_{\text {ins }}}{\text { Sode }}$ & $\frac{P_{1 n y}}{V_{e t}}$ & Circuit & Nodes & Nets & Pins & $\frac{P_{\text {ins }}}{I_{\text {iode }}}$ & $\frac{P_{i n y}}{\text { Iet }^{\prime}}$ \\
\hline $\mathrm{bm01}$ & 12752 & 1.1111 & 50566 & 3.97 & 3.58 & ibm10 & 69.129 & 75196 & 297567 & 4.20 & 3.96 \\
\hline bmo2 & 19601 & 19584 & 81199 & 4.14 & 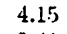 & ibm11 & 70558 & 1454 & 280786 & 8 & 3.45 \\
\hline $\mathrm{ibm03}$ & 23136 & $27+01$ & 93573 & 4.07 & 3.41 & ibm12 & 71070 & 77240 & 317760 & 4.47 & 4.11 \\
\hline$b m 04$ & 27507 & 31970 & 1058550 & 3.85 & 3.31 & ibm13 & 84199 & $9 ! 96(6) 6$ & $357(17$ & 4.24 & 3.58 \\
\hline ibm05 & $293+7$ & 28446 & 126308 & 4.30 & 4.44 & ibm14 & 14760.5 & 152772 & 546816 & 3.70 & 3.58 \\
\hline ibm06 & 32.498 & 34826 & 128182 & $3.9-1$ & 3.68 & ibm 15 & 161.570 & 1866088 & 715823 & 4.13 & 3.84 \\
\hline ibm07 & 45926 & 48117 & 175639 & 3.8 .2 & 3.65 & $i b \log$ & 183484 & $1900+8$ & 778823 & 4.24 & 4.10 \\
\hline $\operatorname{bm} 08$ & 51309 & 50513 & 204890 & 3.99 & 4. & ibm17 & 18.549 .5 & 180581 & 8600.36 & 4.64 & 4.54 \\
\hline ibmog & 533395 & $60(x) 2$ & 222084 & 4.16 & 3.65 & $i b m 18$ & $21061: 3$ & 201920 & 819697 & 3.8!) & 4.06 \\
\hline
\end{tabular}

Table 1.2 The 1998 ISPD Benchmark Circuits

\begin{tabular}{|c|c|c|c|c|c|c|c|}
\hline \multirow[t]{2}{*}{ Circuit } & \multicolumn{3}{|c|}{ Grid } & \multirow{2}{*}{$\begin{array}{c}\text { Average } \\
\text { Wirelength } \\
\sum\left|S^{*}\right|\end{array}$} & \multirow[t]{2}{*}{$\begin{array}{l}\text { Standard } \\
\text { Deviation }\end{array}$} & \multirow{2}{*}{$\begin{array}{l}\text { Minimum } \\
\text { Wirelength } \\
\sum\left|S^{*}\right|\end{array}$} & \multirow[t]{2}{*}{$\begin{array}{l}\text { Runtime } \\
\text { (s) }\end{array}$} \\
\hline & $n_{1}$ & $n_{2}$ & $n_{3}$ & & & & \\
\hline $19 \mathrm{ks}$ & 15 & 14 & 14 & 14493.3 & $1.61 \%$ & 14123 & 37 \\
\hline avq. large & 30 & 30 & 28 & 104104.1 & $1.22 \%$ & 101693 & 303 \\
\hline avq.small & 28 & 28 & 28 & 94688.0 & $0.88 \%$ & 93823 & $2 \pi 7$ \\
\hline balup & 10 & 9 & 9 & 3263.5 & $1.77 \%$ & 3164 & 13 \\
\hline biomedP & 19 & 19 & 19 & 25239.2 & $0.80 \%$ & 248.39 & 106 \\
\hline golem3 & 48 & 48 & 45 & 687104.9 & $0.68 \%$ & 6795.54 & 1519 \\
\hline industry2 & 24 & 23 & 23 & 78997.7 & $0.92 \%$ & 78052 & 202 \\
\hline industry 3 & 26 & 25 & 24 & 152962.3 & $1.0 .5 \%$ & 149592 & 301 \\
\hline $\mathrm{p} 1$ & 10 & 10 & 9 & 4156.1 & $2.17 \%$ & 4071 & 14 \\
\hline p2 & 15 & 15 & 14 & 18562.5 & $1.64 \%$ & 18044 & 43 \\
\hline $\mathbf{s 1 3 2 0 7 P}$ & 21 & 21 & 20 & 26501.3 & $2.15 \%$ & 25617 & 103 \\
\hline s15850P & 22 & 22 & 22 & 30950.7 & $0.65 \%$ & 30729 & 122 \\
\hline s35932 & 27 & 26 & 26 & 57926.1 & $1.74 \%$ & 56120 & 218 \\
\hline s38417 & 29 & 29 & 29 & 73282.6 & $1.21 \%$ & 723555 & 253 \\
\hline s38584 & 28 & 28 & 27 & 72643.9 & $1.10 \%$ & 71560 & 258 \\
\hline s9234P & 19 & 18 & 18 & 17670.1 & $0.89 \%$ & 17463 & 86 \\
\hline structP & 13 & 13 & 12 & 7064.1 & $1.41 \%$ & 6879 & 23 \\
\hline t2 & 12 & 12 & 12 & 8.501 .9 & $1.44 \%$ & 8337 & 22 \\
\hline t3 & 12 & 12 & 12 & 7828.2 & $1.42 \%$ & 7658 & 22 \\
\hline t4 & 12 & 12 & 11 & 7375.9 & $1.63 \%$ & 7242 & 22 \\
\hline t5 & 14 & 14 & 14 & 12568.2 & $0.57 \%$ & 12481 & 36 \\
\hline t6 & 13 & 12 & 12 & 7968.1 & $1.51 \%$ & 7785 & 23 \\
\hline
\end{tabular}

Table 1.3 3-D Placement Results for the ACM/SIGDA Circuit Suite 


\begin{tabular}{|c|c|c|c|c|c|c|c|}
\hline \multirow[t]{2}{*}{ Circuit } & \multicolumn{3}{|c|}{ Grid } & \multirow{2}{*}{$\begin{array}{c}\text { Average } \\
\text { Wirelength } \\
\sum\left|S^{*}\right|\end{array}$} & \multirow[t]{2}{*}{$\begin{array}{l}\text { Standard } \\
\text { Deviation }\end{array}$} & \multirow{2}{*}{$\begin{array}{c}\text { Minimum } \\
\text { Wirelength } \\
\sum\left|S^{*}\right|\end{array}$} & \multirow[t]{2}{*}{$\begin{array}{l}\text { Runtime } \\
\text { (s) }\end{array}$} \\
\hline & $n_{1}$ & $n_{2}$ & $n_{3}$ & & & & \\
\hline ibm01 & 24 & 24 & 2.3 & 92601.5 & $0.91 \%$ & 90591 & 239 \\
\hline ibm02 & 28 & 28 & 26 & 202121.7 & $0.65 \%$ & 199264 & 391 \\
\hline $\mathrm{ibm03}$ & 30 & 30 & 27 & $2: 34600.9$ & $0.94 \%$ & 231824 & $43: 3$ \\
\hline ibm04 & 32 & 30 & 29 & 301324.1 & $1.07 \%$ & 297370 & 497 \\
\hline ibm05 & 32 & 32 & 30 & 367004.5 & $1.27 \%$ & 3606.59 & 5.54 \\
\hline ibm06 & 32 & 32 & 32 & 333985.0 & $3.21 \%$ & 323919 & 655 \\
\hline ibm07 & 36 & 36 & 36 & 473844.3 & $1.82 \%$ & 458047 & 1084 \\
\hline ibm08 & 38 & 38 & 36 & 531860.7 & $1.38 \%$ & 521203 & 1191 \\
\hline ibm09 & 38 & 38 & 37 & 617201.7 & $2.56 \%$ & 587107 & 1263 \\
\hline ibm10 & 42 & 41 & 41 & 832125.1 & $3.79 \%$ & 796763 & 1761 \\
\hline ibm11 & 42 & 41 & 41 & $87^{\circ} 2118.2$ & $2.72 \%$ & 842491 & 1661 \\
\hline ibm12 & 42 & 42 & 41 & 991783.9 & $1.63 \%$ & 959567 & 1864 \\
\hline ibm13 & 44 & 44 & 44 & 1000941.8 & $2.05 \%$ & 971330 & 1864 \\
\hline ibm14 & 54 & 54 & 52 & 1657408.1 & $1.12 \%$ & $16: 30502$ & 3064 \\
\hline ibm15 & 56 & 56 & 54 & 199468.5 .8 & $1.38 \%$ & 1957427 & 3769 \\
\hline ibm16 & 58 & 58 & 57 & 2222138.0 & $1.05 \%$ & 2190510 & 4030 \\
\hline ibm17 & 58 & 58 & 57 & 2745042.7 & $1.16 \%$ & 2680986 & 4463 \\
\hline ibm18 & 60 & 60 & 59 & $26: 39356.6$ & $4.29 \%$ & $250408: 3$ & 428.5 \\
\hline
\end{tabular}

Table 1.4 3-D Placement Results for the ISPD98 Circuit Suite

suite is more established, it lacks the larger circuits that can be found in the ISPD98 suite.

Since the hMetis partitioner is randomized, we perform 10 runs for each circuit on a Pentium II/300MHz system. The results are summarised in tables 1.3 and 1.4. The runtimes indicate the runtime for one run.

\subsection{FROM 2 TO 3 DIMENSIONS}

Besides having a reference platform for 3-D placement wirelengths, it is of interest to know what kind of improvement can be expected from 3D VLSI. In table 1.5 we have compiled wirelength results for four circuits covcring the dynamic range from 800 to 210,000 nodes. For cach circuit we computed a placement in $d=2,21 / 3,22 / 3$, and 3 dimensions. By this we mean that the base of the gate array has an edge length of $\sqrt[d]{N}$. Thus a 2-D gatc array, is of sizc $\sqrt{N} \times \sqrt{N} \times 1$, and a $3-\mathrm{D}$ gate array of size $\sqrt[3]{N} \times \sqrt[3]{N} \times \sqrt[3]{N}$. In gencral, for dimension $d$ and an $N$-node circuit, we selected a grid size close to $\sqrt[d]{N} \times \sqrt[d]{N} \times N /(\sqrt[d]{N})^{2}$. 


\begin{tabular}{|c|c|c|c|c|c|c|c|c|c|}
\hline \multirow[t]{2}{*}{ Circuit } & \multirow[t]{2}{*}{ Nodes } & \multicolumn{3}{|c|}{ Grid } & \multirow{2}{*}{$\begin{array}{c}\text { Dimen- } \\
\text { sions } \\
d\end{array}$} & \multicolumn{2}{|c|}{$\begin{array}{l}\text { All Nets } \\
\text { Counted }\end{array}$} & \multicolumn{2}{|c|}{$\begin{array}{l}\text { 2- and 3-node } \\
\text { Nets Only }\end{array}$} \\
\hline & & $n_{1}$ & $n_{2}$ & $n_{3}$ & & $\begin{array}{l}\text { Length } \\
\sum\left|S^{*}\right|\end{array}$ & Change & $\begin{array}{l}\text { Length } \\
\sum\left|S^{*}\right|\end{array}$ & Change \\
\hline \multirow[t]{4}{*}{ p1 } & 833 & 29 & 29 & 1 & 2 & 6751.5 & & 3800.0 & \\
\hline & & 17 & 17 & 3 & $21 / 3$ & 4584.2 & $-32.1 \%$ & 2727.5 & $-28.2 \%$ \\
\hline & & 12 & 12 & 6 & $22 / 3$ & 4175.2 & $-38.2 \%$ & 2505.5 & $-34.1 \%$ \\
\hline & & 10 & 10 & 9 & 3 & 4143.5 & $-38.6 \%$ & 2498.8 & $-34.2 \%$ \\
\hline \multirow[t]{4}{*}{ s9234P } & 5866 & 78 & 77 & 1 & 2 & 28353.2 & & 17515.8 & \\
\hline & & 40 & 38 & 4 & $21 / 3$ & 19192.9 & $-32.3 \%$ & 13019.4 & $-25.7 \%$ \\
\hline & & 26 & 26 & 9 & $22 / 3$ & 17891.6 & $-36.9 \%$ & 12598.2 & $-28.1 \%$ \\
\hline & & 19 & 18 & 18 & 3 & 17640.6 & $-37.8 \%$ & 12404.3 & $-29.2 \%$ \\
\hline \multirow[t]{4}{*}{ ibm06 } & 32498 & 184 & 180 & 1 & 2 & 792670.2 & & 224425.6 & \\
\hline & & 82 & 82 & 5 & $21 / 3$ & 410851.0 & $-48.2 \%$ & 125969.6 & $-43.9 \%$ \\
\hline & & 50 & 50 & 13 & $22 / 3$ & 372507.4 & $-53.0 \%$ & 133579.7 & $-40.5 \%$ \\
\hline & & 32 & 32 & 32 & 3 & 331429.9 & $-58.2 \%$ & 116558.5 & $-48.1 \%$ \\
\hline \multirow[t]{4}{*}{ ibm 18} & 210613 & 460 & 460 & 1 & 2 & 7705843.6 & & 2561197.9 & \\
\hline & & 188 & 188 & 6 & $21 / 3$ & 3597492.0 & $-53.3 \%$ & 1258260.2 & $-50.9 \%$ \\
\hline & & 98 & 98 & 22 & $22 / 3$ & 2932640.5 & $-61.9 \%$ & 1086833.2 & $-.57 .6 \%$ \\
\hline & & 60 & 60 & 59 & 3 & 2641617.1 & $-6.5 .7 \%$ & 969398.1 & $-62.2 \%$ \\
\hline
\end{tabular}

Table 1.5 Wirelength Improvement from Two to Three Dimensions

Besides the usual total wirelength estimate, table 1.5 also shows the total wirclengths of nets with 2 and 3 nodes. For such nets, the semiperimeter bounding box approximation of the wirelength is exact.

We notice that a substantial wirelength advantage can be achieved for large circuits even when only a few layers are employed. While small circuits exhibit only a modest wirelength improvement in 3-D, larger circuits clearly benefit from the third dimension. Small circuits $\mathrm{p} 1$ and s9234P experience only a $30 \%-40 \%$ improvement in a $3-\mathrm{D}$ placement, whereas ibm18 saves approximately $50 \%$ wirelength in $21 / 3$ dimensions and over $60 \%$ in 3 dimensions.

\section{CONCLUSION}

We presented a general definition of the 3-D placcment problem. This definition can be used to compare 3-D placement algorithms to each other. On the basis of this definition, we implemented a partitioning placer and generated a first set of comprehensive wirelength results for 3-D placements of circuits in two benchmark circuits suites.

Further, we compared wirelength results of selected circuits for placements varying from two to three dimensions. These results provide empirical evidence that large circuits benefit substantially from utilising 
the third dimension even if only a small number of layers in the third dimension is used.

\section{Acknowledgments}

We thank Prof. Karypis for making the h-Tetis 1.5.3 library available and providing support for its use.

\section{References}

Alpert, C. J. (1998). The ISPD98 circuit benchmark suite. In Proceedings of the International Symposium on Physical Design, pages 85-90.

Brglez, F. (1993). ACM/SIGDA design automation benchmarks: Catalyst or anathoma? IEEE Design \&3 Test of Computers, 10(3):87-91.

Chiricescu, S. M. S. A. and Vai, M. M. (1998). A three-dimensional FPGA with an integrated memory for in-application reconfiguration data. In 1998 IEEE International Symposium on Circuits and Systems, volume 2, pages 232-235.

Depreitere, J., Neefs, II., Van Marck, II., Van Campenhout, J., Baets, R., Dhoedt, B., Thienpont, II., and Veretennicoff, I. (1994). An optoelectronic: 3-d field programmable gate array. In Field-programmable logic : architectures, synthesis, and applications : 4th International Workshop on Field-Programmable Logic and Applications, volume 849 of Lecture notes in computer science, pages 352-360.

IIanan, M. (1966). On Stciner's problem with rectilinear distancc. SIAM Journal on Applied Mathematics, 14(2):255-265.

Karypis, G. (1999). private communication.

Karypis, G., Aggarwal, R., Kumar, V., and Shckhar, S. (1997). Multilevel hypergraph partitioning: Application in VLSI domain. In Proceedings of the 34th ACM/IEEE Design Automation Conference, pages $526-529$.

Leeser, M., Melcis, W. M., Vai, M. M., Chiricescu, S., Xu, W., and Zavracky, P. M. (1998). Rothko: A threc-dimensional FPGA. IEEE Designs and Test of Computers, 15(1):16-23.

Leighton, F. T. and Roscnberg, A. L. (1986). Three-dimensional circuit layouts. SIAM Journal on Computing, 15(3):793-813.

Ohmura, M. (1998). An initial placement algorithm for 3-d VLSI. In 1998 IEEE International Symposium on Circuits and Systems, volume 6, pages 195-198.

Shahookar, K. and Mazumder, P. (1991). VLSI cell placement techniques. ACM Computing Surveys, 23(2):143-220.

Tong, C. C. and Wu, C. (1995). Routing in a three-dimensional chip. IEEE Transactions on Computers, 44(1):106-117. 